\title{
An Improvement on Codebook Search for Vector Quantization
}

\author{
L.Torres J.Huguet
}

\begin{abstract}
We present a simple but yet effective algorithm to speed up the codebook search in a vector quantization scheme when a MSE criterium is used. A considerable reduction in the number of operations is achieved. This algorithm was originally designed for image vector quantization in which the samples of the image signal (pixels) are positive, although it can be used with any positivenegative signal with only minor modifications.
\end{abstract}

\section{INTRODUCTION}

The vector quantization scheme has proven to be very effective in speech and image coding [1], where a number of samples are grouped together forming a so called vector. Each vector $X$ is then treated as an unbreakable unit during the encoding/decoding operation.

In image vector quantization, the image is divided into small rectangular blocks typically of size $3 \times 3$ or $4 \times 4$ pixels or similar. Although in this case $X$ has the structure of a matrix rather than of a vector we prefer to call it vector in a general sense as this is the most common terminology. Let $X$ be an input vector from the original signal and let $Y$ represent a codeword or reproduction vector. The codebook contains all the available codewords and the quantizer replaces the input vector by the codeword that is more similar to $X$. If the usual minimum square error is used as the measure of similarity, the quantizer searches for the codeword located at the minimum error from the input vector. Other distortion measures are also used, although will not be considered in this work. In order to assign to $X$ the best codeword of the codebook, an exhaustive search (also called full search) is required. That means that every codeword has to be examined to ensure that the best will be selected. When the number of codewords is large this process requires a very important computational load. Several algorithms $[2,3,4]$ have been designed to reduce the computational load of the encoding process with the same goal as the one presented here, i.e. to reduce the computational task.

Paper approved by Farokh Marvasti, the Editor for Communications of the IEEE Communications Society. Manuscript received May 6, 1991; revised April 15, 1992. This paper was supported by PRONTIC Grant $105 / 88$ of the Spanish Government, and was partially presented at the Visual Communications and Image Processing'90 Conference, October 1990, Lausanne, Switzerland.

The authors are with the Department of Signal Theory and Communications. Polytechnical University of Catalonia, Barcelona, Spain.

\section{THE ALgORITHM}

The algorithm we present reduces the encoding complexity when the minimum square error is used to evaluate the similarity between the signal vector and the codeword. This is the most widely used distortion measure in speech and image coding due to its good results and simplicity.

The distortion between the vector $X=\left(x_{1}, \ldots, x_{N}\right)$ and the codeword $Y=\left(y_{1}, \ldots, y_{N}\right)$ is computed as follows

$$
d(X, Y)=\sum_{n=1}^{N}\left(x_{n}-y_{n}\right)^{2}
$$

to which we will refer as the "real distortion". It is clear that a distortion calculation implies $N$ multiplications and $(2 N-1)$ additions/subtractions. The multiplication operation is usually taken as the most processor time consuming factor and has become the standard measure when evaluating the complexity of an algorithm.

Equation (1) can be developed as follows

$$
\begin{aligned}
d(X, Y) & =\sum_{n=1}^{N} x_{n}^{2}+\sum_{n=1}^{N} y_{n}^{2}-2 \sum_{n=1}^{N} x_{n} y_{n}= \\
& =\|X\|^{2}+\|Y\|^{2}-2 \sum_{n=1}^{N} x_{n} y_{n}
\end{aligned}
$$

$\|Y\|^{2}$ can be previously calculated and stored to be used every time the distortion between a vector and a codeword has to be computed. On the other hand, there is no need to compute $\|X\|^{2}$ as it depends only on the input vector $X$ and for a given vector it is a constant that does not affect the nearest neighbor selection.

The term $\sum x_{n} y_{n}$ depends on both the vector and the codeword and cannot be precalculated as before. However, if all the vector components are positive, i.e., $x_{n} \geq 0$ and $y_{n} \geq 0$ then it is obvious that

$$
\sum_{n=1}^{N} x_{n} y_{n} \leq x_{\max } \sum_{n=1}^{N} y_{n}
$$


where $x_{\text {max }}$ is the maximum vector component of vector $X$, that is $x_{\max }=\max \left(x_{1}, \ldots, x_{N}\right)$.

With that idea in mind we can define the following distortion

$$
d_{l}(X, Y)=\|X\|^{2}+\|Y\|^{2}-2 x_{\max } \sum_{n=1}^{N} y_{n}
$$

which always satisfies

$$
d_{l}(X, Y) \leq d(X, Y)
$$

The computation of $d_{l}(X, Y)$ is very simple (1 multiplication and 2 additions) if we assume that $\|Y\|^{2}, x_{\max }$ and $2 \Sigma y_{n}$ have been previously calculated and stored. A description of the proposed algorithm is given as follows

Evaluate and store $\|Y\|^{2}$ of every codeword Evaluate and store $2 \sum_{n=1}^{N} y_{n}$ of every codeword

for $i=1$ to $I$

Evaluate $x_{\max }$

distmin $=\infty$

for $j=1$ to $J$ dist $=d_{1}\left(X_{i}, Y_{j}\right)$;

if (dist $>$ distmin) continue ;

dist $=d\left(X_{i}, Y_{j}\right)$;

if (dist $>$ distmin) continue ;

index $=j ; \quad$ (codeword selected $Y_{j}$ )

distmin $=$ dist;

next $j$

next $i$

The continue statement indicates that the current iteration in the loop has finished so the next iteration can start. This algorithm is called "the single test algorithm". The algorithm calculates $d_{l}\left(X_{i}, Y_{j}\right)$ with little computational work as an estimation of the real distortion $d\left(X_{i}, Y_{j}\right)$, and in the case that $d_{l}\left(X_{i}, Y_{j}\right)>$ distmin there is no need to calculate the real distortion as it will be true that $d\left(X_{i}, Y_{j}\right)>$ distmin as indicated by (5). Then, in this case, codeword $Y_{j}$ may be discarded. That means that if the test succeeds we save the calculation of the real distortion what would imply many more operations.

The efficiency of the algorithm depends mainly on the number of times the test above explained succeeds. As an alternative, equation (4) could have been taken as

$$
d_{2}(X, Y)=\|X\|^{2}+\|Y\|^{2}-2 y_{\max } \sum_{n=1}^{N} x_{n}
$$

and it is difficult to say which is the best alternative as it depends on the particular values of the vectors and codewords.
It is also possible to use both distortions such that if the first test fails then the second distortion $d_{2}$ may be calculated. If it also fails then the real distortion has to be calculated. This combination, which gives the fastest encoding algorithm of all tested is implemented as follows

Evaluate and store $\|\left. Y\right|^{2}$ of every codeword

Evaluate and store $2 \sum_{n=1}^{N} y_{n}$ and $y_{\max }$ of every codeword for $i=I$ to $I$

Evaluate $x_{\max }$ and $2 \sum_{n=1}^{N} x_{n}$

distmin $=\infty$

for $j=l$ to $J$

dist $=d_{l}\left(X_{i}, Y_{j}\right)$;

if (dist > distmin) continue;

dist $=d_{2}\left(X_{i}, Y_{j}\right)$;

if (dist $>$ distmin) continue;

dist $=d\left(X_{i}, Y_{j}\right)$;

if (dist $>$ distmin) continue;

index $=j ; \quad$ (codeword selected $Y_{j}$ )

distmin $=$ dist;

next $i$ next $j$

This algorithm is called "the double test algorithm". A further improvement has been added to our method by combining it with the "partial distortion" method introduced in $[3,4]$. The "partial distortion" calculates the euclidean distortion of (1) but after each accumulation it checks if the partially calculated distortion is greater than the distortion to the best codeword found so far (variable distmin in our algorithms). So, if the test in (5) or (6) fails and the real distortion has to be calculated, equation (1) is found via the "partial distortion".

One of the limitations of the algorithm is that it requires that the vector components $x_{n}$ and $y_{n}$ must be positive. For positive and negative vector components (3) is not correct. Negative vector components may occur, for instance, in Image Transform Vector Quantization encoding schemes or in Speech Coding. One solution to this problem is to add a positive offset so that all vector components become positive. If $p$ is the most negative sample of $X$ and $Y\left(p=\min \left(x_{1}, \ldots, x_{N}\right.\right.$, $\left.y_{1}, \ldots, y_{N}\right)$ ) then we can modify the vector components as follows

$$
\begin{aligned}
& x_{n}^{\prime}=x_{n}+|p| \quad n=1, \ldots, N \\
& y_{n}^{\prime}=y_{n}+|p| \quad n=1, \ldots, N
\end{aligned}
$$

It is easy to verify that the real distortion between the modified vector and the codewords remains the same

$$
d\left(X^{\prime}, Y^{\prime}\right)=\sum_{n=1}^{N}\left(x_{n}^{\prime}-y_{n}^{\prime}\right)^{2}=d(X, Y)
$$




\section{RESULTS}

We have performed a variety of experiments to show the goodness of the proposed algorithm. The well known 256x256 images Lenna, Walter and Miss America have been selected as test images. Three different codebook sizes, 256, 512 and 1024 have been chosen and the images have been divided in square subimages of $3 \times 3$ pixels for vector quantization coding. All the codebooks were designed using the LBG algorithm [5] although the performance of our method does not depend on how the codebook is generated but rather on the structure of the vector components used to generate the codebook. The proposed single test algorithm has been compared against the "partial distortion" algorithm and against the euclidean distortion to evaluate its performance. The results are given in computer time percentage required to code the above explained images using the standard full search VQ coding scheme. All the algorithms have been run on a IBM-RT 6150 .

TABLE 1. RESULTS IN CPU TIME REQUIRED TO VQ ENCODING images LenNa, WaLter and Miss AMERICA USING THE EUCLIDEAN DISTORTION (E.D.) THE "PARTIAL DISTORTION" (P.D.) AND THE SINGLE TEST (S.T.) ALGORITHM.

\begin{tabular}{|c|ccc|ccc|ccc|}
\hline IMAGE & \multicolumn{3}{|c|}{ LENNA } & \multicolumn{3}{c|}{ WALTER } & \multicolumn{3}{c|}{ MISS AMERICA } \\
\hline Codebook & 256 & 512 & 1024 & 256 & 512 & 1024 & 256 & 512 & 1024 \\
\hline E. D. & 100 & 100 & 100 & 100 & 100 & 100 & 100 & 100 & 100 \\
\hline P. D. & 45.9 & 43.46 & 42.46 & 44.4 & 42.93 & 1.54 & 51.1 & 48.85 & 46.98 \\
\hline S. T. & 38.3 & 36.16 & 34.35 & 31.9 & 29.64 & 7.69 & 45.6 & 51.66 & 2.01 \\
\hline
\end{tabular}

All the results using the single test algorithm show a decrease in computer time required to code the images with respect to the full search method. This decrease depends on the image but can be stated in about 10 per cent. A further improvement can be obtained if the single test algorithm is combined with the "partial distortion" algorithm or the double test algorithm is combined with the "partial distortion" algorithm. These improvements can be stated in about 5 per cent savings with respect to the single test algorithm.

\section{Conclusions}

A new fast algorithm for image quantization codebook search, when the minimum square error is the distortion measure, has been introduced. The results show a reduction in the number of additions and multiplications when compared against other well known techniques.

The efficiency and simplicity of the algorithm presented to accelerate the encoding process in a vector quantization scheme, makes it highly attractive for image quantization where a large amount of data has to be processed. The algorithm can be easily incorporated into a working system by simply adding the "single test" or "double test" algorithms.

\section{REFERENCES}

[1] R.M. Gray, "Vector quantization," IEEE ASSP Magazine, vol. 1, April 1984.

[2] M.R. Soleymani and S.D. Morgera. "A fast encoding technique for vector quantization," IEEE Trans. Commun., vol. COM-37, June 1989.

[3] Chang-Da Bei and R.M. Gray, "An improvement of the minimum distortion encoding algorithm for vector quantization," IEEE Trans. Commun., vol. COM-33, October 1985.

[4] D.Y. Cheng, A. Gersho, B. Ramamurthi and Y. Shoham, "Fast search algorithms for vector quantization and pattern matching," Proc. International Conference on Acoustics Speech and Signal Processing, vol. 1, San Diego, USA, March 1984.

[5] Y. Linde, A. Buzo and R.M. Gray, "An algorithm for vector quantizer design," IEEE Trans. Commun., vol. COM- 28, Jan. 1980 